\title{
Case Report \\ Primary Pericardial Sarcoma: A Case Report and a Brief Review
}

\author{
Aneesh Pakala, ${ }^{1}$ Raghav Gupta, ${ }^{2}$ and Ralph Lazzara ${ }^{2}$ \\ ${ }^{1}$ Department of Internal Medicine, The University of Oklahoma Health Sciences Center, Williams Pavillion 2040, \\ P.O. Box 26901, Oklahoma City, OK 73190, USA \\ ${ }^{2}$ Department of Cardiovascular Medicine, The University of Oklahoma Health Sciences Center, Oklahoma City, OK 73190, USA
}

Correspondence should be addressed to Aneesh Pakala, aneesh-pakala@ouhsc.edu

Received 30 September 2010; Revised 20 January 2011; Accepted 25 February 2011

Academic Editor: Syed Wamique Yusuf

Copyright () 2011 Aneesh Pakala et al. This is an open access article distributed under the Creative Commons Attribution License, which permits unrestricted use, distribution, and reproduction in any medium, provided the original work is properly cited.

There are very few cases of primary pericardial sarcomas reported in the English literature. Pericardial tumors, like other cardiac tumors, are most likely to be metastatic in nature and are an extension of primary tumors from the surrounding structures. Sarcomas are the most common primary pericardial tumors. Surgical eradication of the tumor is considered to be the treatment of choice. We are presenting a case of a primary pericardial, high-grade pleomorphic undifferentiated sarcoma that was diagnosed at our institution. We discuss the available diagnostic modalities and also shed light on alternative therapies when patients are not ideal surgical candidates.

\section{Introduction}

Cardiac neoplasms are either primary or secondary; in case of secondary neoplasms the most common site of origin is the lung [1]. The cardiac involvement can be endocardial, myocardial, or pericardial, but tumors only involving the parietal pericardium are not considered to be true cardiac tumors. Primary cardiac tumors are rare; the incidence is reported to be around $0.056-0.02 \%$ [1]. Malignant tumors involving the pericardium are even rarer in autopsy series, the incidence is reported to be around $0.001 \%$. Pericardial tumors, like other cardiac tumors, are most likely to be metastatic in nature and are an extension of primary tumors from the surrounding structures.

Pericardial tumors often cause symptoms related to malignant pericardial effusion. The presenting symptoms are cough, dyspnea, chest pain, and palpitations, which are a result of the mass effect of the tumor on the cardiac chambers [2]. These tumors are of a particular concern due to the fact that overt signs and symptoms occur rather late in the course, precluding effective tumor eradication [2]. We are presenting a case of a primary pericardial, high-grade pleomorphic undifferentiated sarcoma that was diagnosed at our institution and provide a brief insight into the detection and treatment of the disease.

\section{Case Presentation}

A 67-year-old Caucasian gentleman presented to our institution with acute right-sided chest pain. He had been experiencing gradually worsening shortness of breath on exertion for a few months. Physical examination revealed a malnourished moderately built male with tachycardia and muffled heart sounds. Chest radiography revealed a widened mediastinum and an enlarged heart that was suspicious for a pericardial effusion. A transthoracic echocardiogram (TTE) confirmed a large anterior pericardial effusion with respirophasic blunting of the inferior vena cava but no right ventricular diastolic collapse (Figure 1). A computed tomography (CT) scan that closely followed the TTE revealed an anterior mediastinal mass arising from the pericardium, compounded by the previously documented pericardial effusion (Figure 2). Pericardial fluid cytology revealed malignant cells. CT guided fine needle aspiration cytology of the pericardial mass was done. Histopathology showed a moderately cellular, spindle cell neoplasm with pleomorphic nuclei in a myxoid background (Figure 3 ). The pathologists did find pseudolipoblasts, which are found in many tumors including pleomorphic liposarcomas (which can be confused with undifferentiated sarcomas [3]). Immunohistochemistry was positive for vimentin, CD163 and CD 68, other muscle 


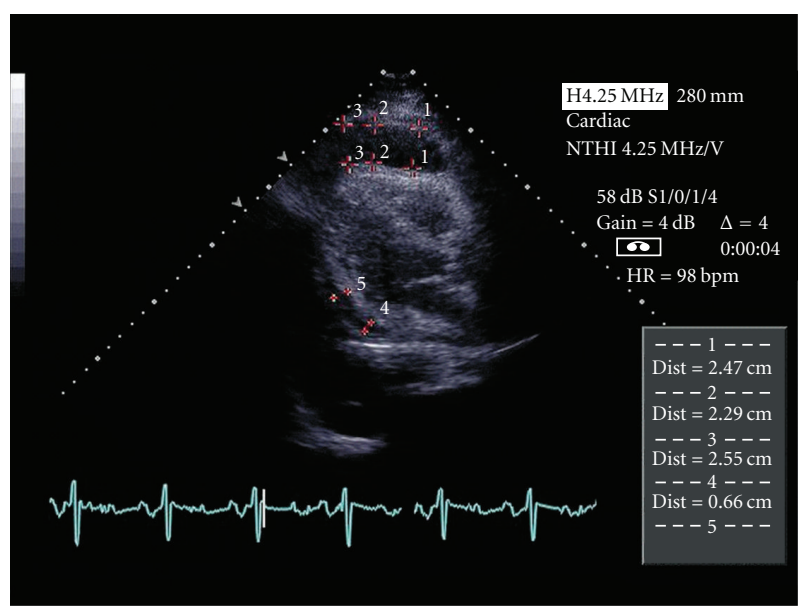

FIGURE 1: Echocardiographic image showing anterior and posterior pericardial effusion.

specific markers but melanocytic markers were negative. The tumor was diagnosed as a primary pericardial high-grade pleomorphic undifferentiated sarcoma.

Surgery is the most effective treatment for sarcoma; however our patient was not considered to be an ideal surgical candidate due to the advanced nature of his disease and poor nutritional state. Oncology was consulted and further treatment options of radiation and chemotherapy were discussed with the patient. Doxorubicin was not considered a feasible option in his case due to its cardiotoxic potential hence a decision was made to attempt a trial of imatinib mesylate and further testing of the pericardial mass for c-kit staining was done. The tumor was found to be c-kit negative suggesting that chemotherapy with imatinib was unlikely to be of benefit and the patient was started on a radiotherapy protocol. Eventually the patient succumbed to the disease in a matter of months.

\section{Pathology}

Sarcomas are the most common primary pericardial tumors; other tumors that involve the pericardium are mesotheliomas, lymphomas, and hemangiomas. Among the sarcomas, undifferentiated sarcomas (previously called malignant fibrous histiocytomas) are most frequent. These are thought to arise from primitive mesenchymal cells and are composed of histiocytic and fibroblastic elements in a storiform pattern. The use of immunohistochemistry, cytogenetics and molecular diagnostics has made it possible to identify other types of sarcomas including myxoid, giant cell, inflammatory, and angiomatoid sarcomas [3, 4]. Undifferentiated sarcomas are further classified into high-grade pleomorphic sarcoma, pleomorphic sarcoma with giant cells, and pleomorphic sarcoma with predominant inflammation on the basis of their histologic appearance [5]. Multiple studies have proved that undifferentiated sarcoma is but a common pathway for developing sarcomas and that a tissue of origin should be actively sought before labeling a soft tissue sarcoma as

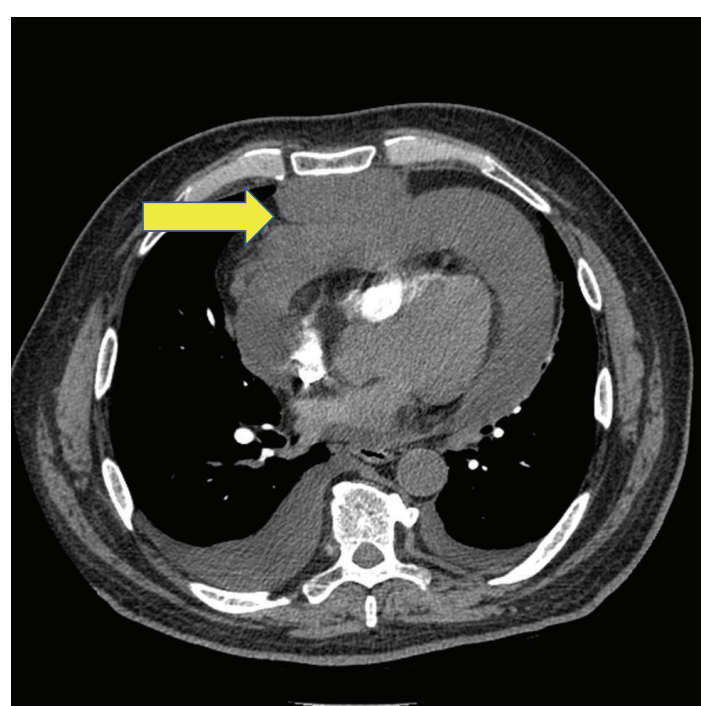

Figure 2: CT showing anterior mediastinal mass arising from the pericardium.

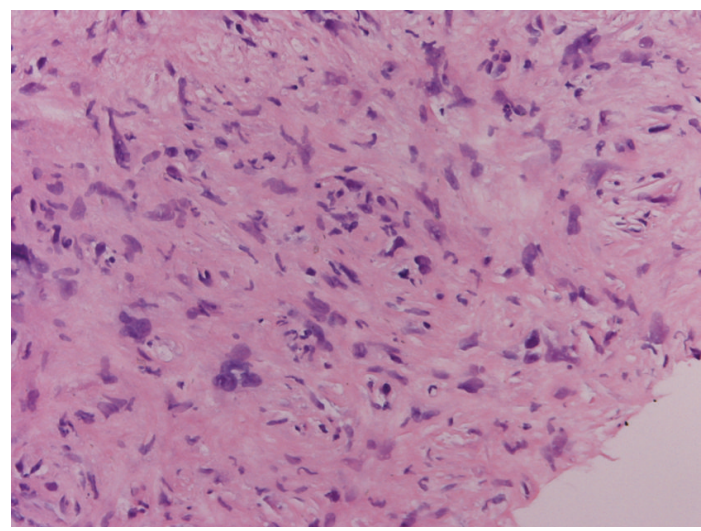

FIGURE 3: Histopathology showing a moderately cellular, spindle cell neoplasm with pleomorphic nuclei in a myxoid background.

an undifferentiated sarcoma $[5,6]$. Pleomorphic undifferentiated sarcomas are commonly seen in elderly males and involve the thigh, upper arm, and the retroperitoneum [3]. We have presented a high-grade pleomorphic undifferentiated sarcoma arising from the pericardium that is very rarely documented in English literature.

\section{Diagnosis}

In case of cardiac sarcomas, a TTE is a good initial diagnostic test to detect the associated pericardial effusion. With the use of contrast echocardiography the sensitivity of detecting malignant tumors increases due to the increased pixel intensity of tumors as compared to the surrounding myocardium [7]. Not all pericardial tumors are associated with pericardial effusion and computed tomography (CT) scan is useful in the detection of such cases. The CT scan finding of pericardial nodularity is highly suggestive of a pericardial tumor [8]. Another distinct advantage over TTE is that CT scan can 
identify the dominant mass and offer guidance for biopsies of the mass [8]. Advanced modalities like multidetector CT scans and cardiac magnetic resonance imaging are gaining favor as the imaging of choice for diagnoses of pericardial sarcomas but they are expensive and their availability is limited [9]. Tissue sampling in the form of surgical biopsies provides the best odds for detection of the cell of origin. Since pleomorphic undifferentiated pattern can be found adjacent to areas of well-differentiated sarcomas in the same tumor, the distinction between pleomorphic undifferentiated sarcomas and other sarcomas can pose a difficult problem for a pathologist [10]. This makes it imperative that every effort should be made to obtain as much tissue as possible to prevent misdiagnoses. Accurate tissue diagnoses have been shown to affect prognosis since myogenic differentiation is considered to be more aggressive than some of the other pleomorphic sarcomas [6]. A surgical biopsy is fraught with periprocedural risks depending on the site of the tumor. We suggest that multiple CT-guided biopsies from different accessible sites should be preferred over open surgical biopsies $[2,6]$.

When such a disease process is suspected, a less invasive procedure like pericardial fluid cytology will provide information regarding the presence of malignancy. It is well accepted that pericardial fluid cytology is indicated in asymptomatic young adults who have an incidental finding of a pericardial effusion. We suggest that pericardial fluid cytology should also be extended to older individuals who do not have an obvious underlying cause for an effusion, in an effort to get a lead time advantage for diagnosing a pericardial malignancy. Positive cytology should be followed by multiple CT-guided biopsies in an effort to make an accurate tissue diagnosis, to guide treatment and also assesses the true prevalence of these tumors [6].

\section{Management}

Surgery is the mainstay of treatment for soft tissue sarcomas and effective tumor removal depends on the anatomic location of the tumor. Pericardial sarcomas may be amenable to removal if detected very early on but our case reflects that this may not be possible due to its covert presentation. The response of soft tissue sarcomas to radiation has been well documented and currently adjuvant radiation is recommended along with surgical resection to improve overall survival $[5,11,12]$.

Chemotherapy is currently reserved for metastatic tumors and the agents of choice are doxorubicin and ifosfamide, with response rates ranging from $55-66 \%[13,14]$. In case of pericardial sarcomas, the use of chemotherapeutics may extend to the treatment of the primary tumor if surgery is ineffective. The use of doxorubicin in pericardial sarcomas may be restricted due to its cardiotoxicity which may confound the mass effects of the tumor and further worsen cardiac reserve. The detection of pericardial tumors should prompt clinicians to enroll these patients into studies that determine the efficacy of novel chemotherapeutics [15]. The tumor should be evaluated for the presence of specific targets (for newer experimental agents) like c-kit, epidermal growth factor receptor, vascular endothelial growth factor receptor, and platelet growth factor receptor, among others $[15,16]$. Gemcitabine has shown promise in the treatment of soft tissue sarcomas that have failed anthracycline-based chemotherapeutic regimen $[17,18]$. Trials with imatinib mesylate have shown some benefit in gastrointestinal soft tissue sarcomas, and ongoing multicenter trials for its use in other soft tissue sarcomas have shown modest results [19]. Further studies are needed to guide chemotherapeutic management.

\section{Prognosis}

Pleomorphic undifferentiated sarcomas in general have a 2year survival rate of $60 \%$ with a high rate of recurrence and metastasis. In a series of 200 cases of undifferentiated sarcomas, the rate of recurrence after primary tumor eradication was around $44 \%$ [ 3 ]. The rate of metastasis was around $42 \%$ and correlated with the depth of the tumor spread, with deeper tumors possessing greater potential for metastasis [3]. The rates for survival, metastasis, and recurrence specific for primary pericardial sarcomas are not available. Given the close proximity of these tumors to vital structures, it is quite possible that mass effect of the tumor itself may be lethal, making metastasis and recurrence less important issues.

\section{Discussion}

The relative paucity of such cases makes it difficult to design management protocols specific to pericardial sarcomas. Detection starts by identification with contrast enhanced TTE, pericardial fluid cytology which should be followed by accurate tissue diagnosis with multisite CT-guided biopsies and cardiothoracic surgery consultation. Currently we believe that surgical approach should be the primary modality of treatment. However if patients are not found to be ideal surgical candidates then chemotherapy and radiation should be offered requiring a multidisciplinary approach by cardiology and oncology. Enrollment in clinical trials involving chemotherapeutic agents should always be considered in this patient population.

\section{Conflict of Interests}

The authors declare no conflict of interests.

\section{Disclaimer}

This paper is not under simultaneous consideration by another journal.

\section{References}

[1] K. A. Ekmektzoglou, G. F. Samelis, and T. Xanthos, "Heart and tumors: location, metastasis, clinical manifestations, diagnostic approaches and therapeutic considerations," Journal of Cardiovascular Medicine, vol. 9, no. 8, pp. 769-777, 2008. 
[2] T. F. Kindl, A. M. Hassan, R. L. Booth Jr., S. J. Durham, and T. J. Papadimos, "A primary high-grade pleomorphic pericardial liposarcoma presenting as syncope and angina," Anesthesia and Analgesia, vol. 102, no. 5, pp. 1363-1364, 2006.

[3] S. W. Weiss and F. M. Enzinger, "Malignant fibrous histiocytoma. An analysis of 200 cases," Cancer, vol. 41, no. 6, pp. 2250-2266, 1978.

[4] M. B. Laya, J. A. Mailliard, C. Bewtra, and H. S. Levin, "Malignant fibrous histiocytoma of the heart. A case report and review of the literature," Cancer, vol. 59, no. 5, pp. 10261031, 1987.

[5] A. F. Nascimento and C. P. Raut, "Diagnosis and management of pleomorphic sarcomas (so-called " MFH") in adults," Journal of Surgical Oncology, vol. 97, no. 4, pp. 330-339, 2008.

[6] A. P. Dei Tos, "Classification of pleomorphic sarcomas: where are we now?” Histopathology, vol. 48, no. 1, pp. 51-62, 2006.

[7] U. C. Rangasetty, J. D. Martinez, and M. Ahmad, "Images in cardiovascular medicine. Contrast-enhanced echocardiography in spindle cell sarcoma of the pericardium," Circulation, vol. 115, no. 11, pp. e329-e331, 2007.

[8] W. B. Dawson, J. R. Mayo, and N. L. Muller, "Computed tomography of cardiac and pericardial tumors," Canadian Association of Radiologists Journal, vol. 41, no. 5, pp. 270-275, 1990.

[9] E. Y. Kim, Y. H. Choe, K. Sung, S. W. Park, J. H. Kim, and Y. H. Ko, "Multidetector CT and MR imaging of cardiac tumors," Korean Journal of Radiology, vol. 10, no. 2, pp. 164-175, 2009.

[10] C. D. M. Fletcher, P. Gustafson, A. Rydholm, H. Willén, and M. Åkerman, "Clinicopathologic re-evaluation of 100 malignant fibrous histiocytomas: prognostic relevance of subclassification," Journal of Clinical Oncology, vol. 19, no. 12, pp. 3045-3050, 2001.

[11] S. A. Rosenberg, J. Tepper, E. Glatstein et al., "The treatment of soft-tissue sarcomas of the extremities. Prospective randomized evaluations of (1) limb-sparing surgery plus radiation therapy compared with amputation and (2) the role of adjuvant chemotherapy," Annals of Surgery, vol. 196, no. 3, pp. 305-315, 1982.

[12] P. W. T. Pisters, L. B. Harrison, D. H. Y. Leung, J. M. Woodruff, E. S. Casper, and M. F. Brennan, "Long-term results of a prospective randomized trial of adjuvant brachytherapy in soft tissue sarcoma," Journal of Clinical Oncology, vol. 14, no. 3, pp. 859-868, 1996.

[13] S. Leyvraz, M. Bacchi, T. Cerny et al., "Phase I multicenter study of combined high-dose ifosfamide and doxorubicin in the treatment of advanced sarcomas. Swiss Group for Clinical Research (SAKK)," Annals of Oncology, vol. 9, no. 8, pp. 877884, 1998.

[14] S. R. Patel, S. Vadhan-Raj, M. A. Burgess et al., "Results of two consecutive trials of dose-intensive chemotherapy with doxorubicin and ifosfamide in patients with sarcomas," American Journal of Clinical Oncology, vol. 21, no. 3, pp. 317321, 1998.

[15] J. T. Hartmann, "Systemic treatment options for patients with refractory adult-type sarcoma beyond anthracyclines," AntiCancer Drugs, vol. 18, no. 3, pp. 245-254, 2007.

[16] J. M. Magenau and S. M. Schuetze, "New targets for therapy of sarcoma," Current Opinion in Oncology, vol. 20, no. 4, pp. 400406, 2008.

[17] J. T. Hartmann, K. Oechsle, J. Huober et al., "An open label, non-comparative phase II study of gemcitabine as salvage treatment for patients with pretreated adult type soft tissue sarcoma," Investigational New Drugs, vol. 24, no. 3, pp. 249 $253,2006$.
[18] M. L. Hensley, R. Maki, E. Venkatraman et al., "Gemcitabine and docetaxel in patients with unresectable leiomyosarcoma: results of a phase II trial," Journal of Clinical Oncology, vol. 20, no. 12, pp. 2824-2831, 2002.

[19] R. Chugh, J. K. Wathen, R. G. Maki et al., "Phase II multicenter trial of imatinib in 10 histologic subtypes of sarcoma using a bayesian hierarchical statistical model," Journal of Clinical Oncology, vol. 27, no. 19, pp. 3148-3153, 2009. 


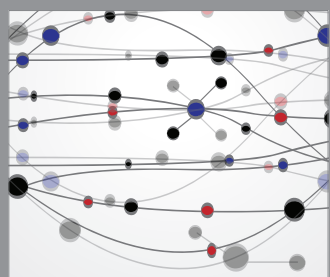

The Scientific World Journal
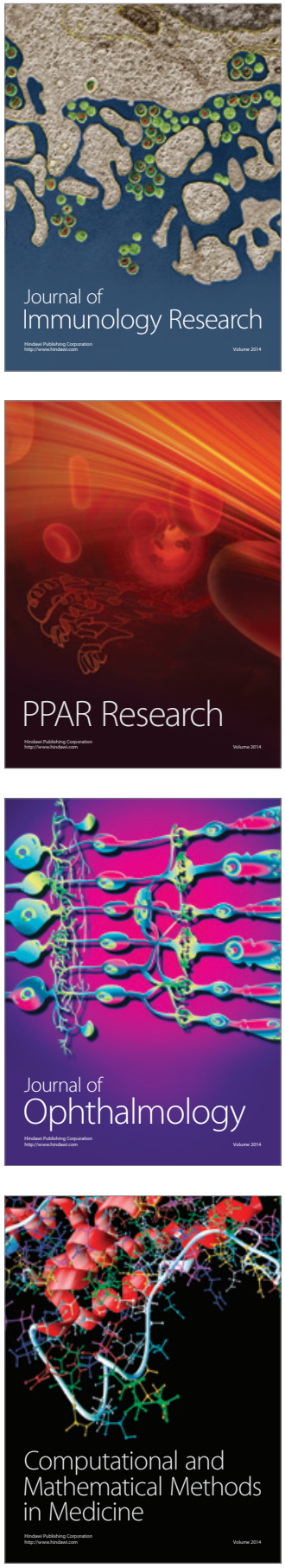

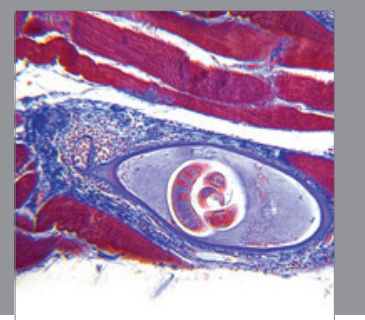

Gastroenterology

Research and Practice
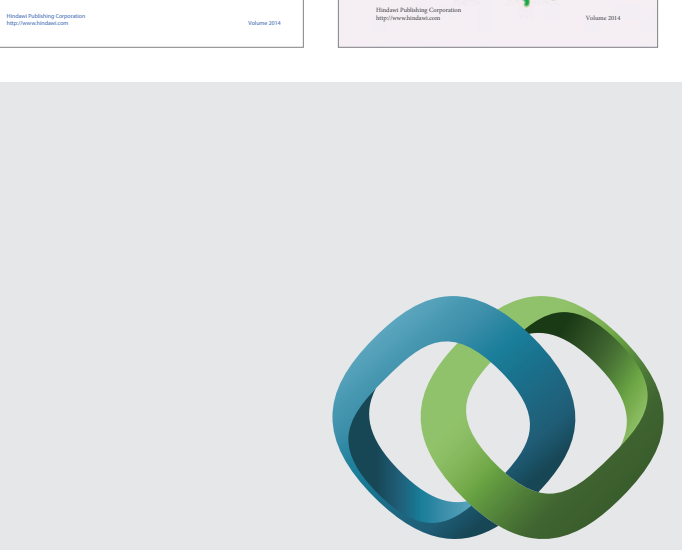

\section{Hindawi}

Submit your manuscripts at

http://www.hindawi.com
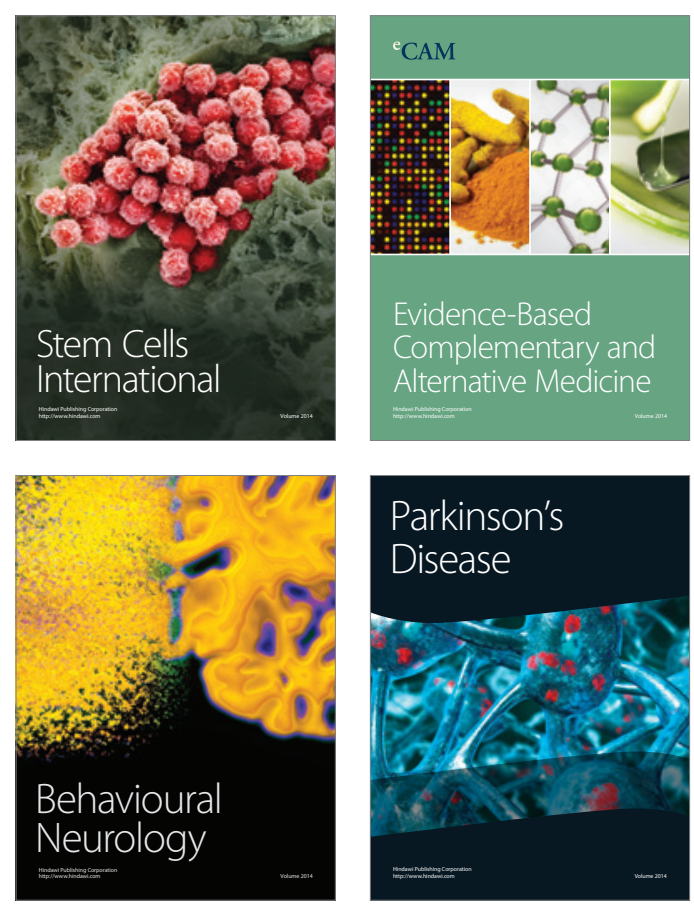

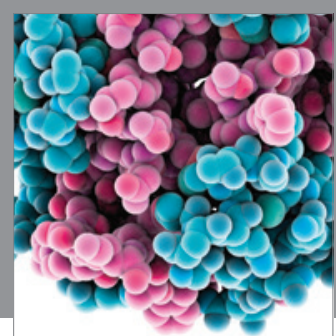

Journal of
Diabetes Research

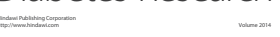

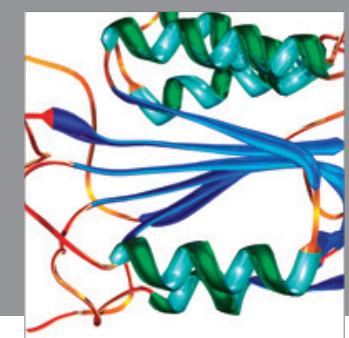

Disease Markers
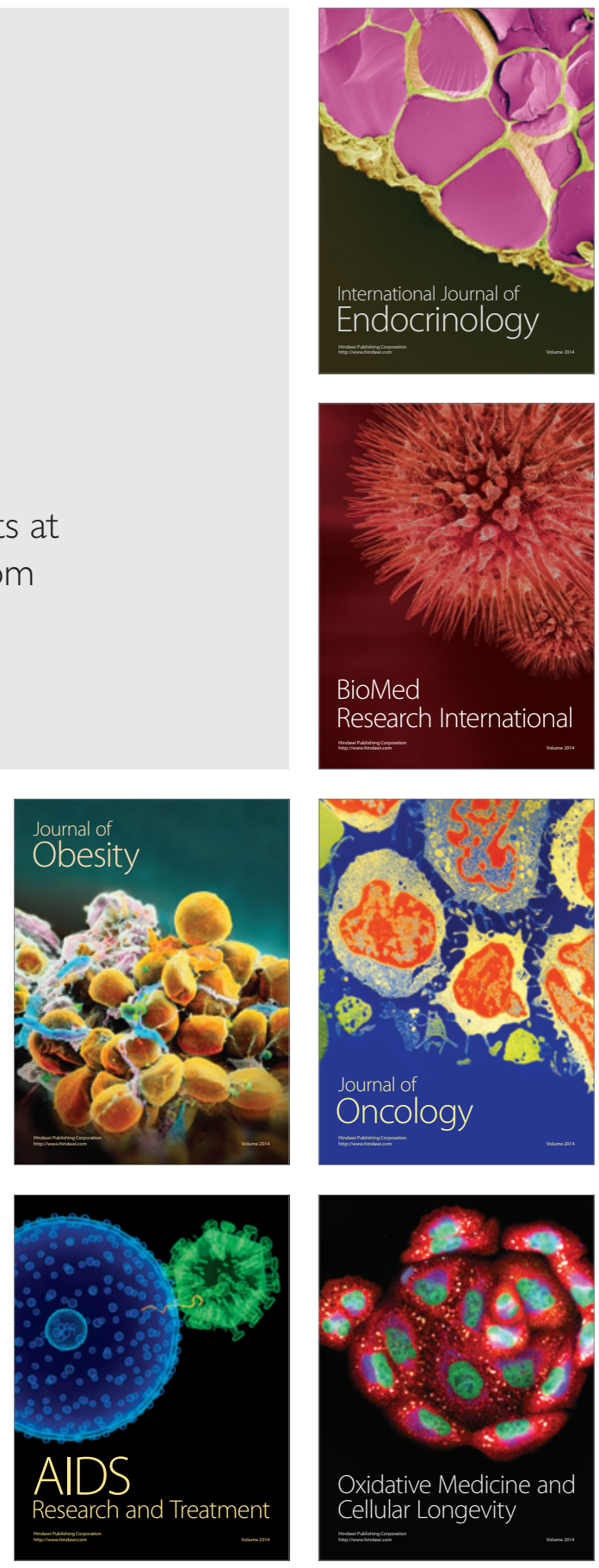